\title{
Histopathological Study of Pure Primary Nephrotic Syndrome in Adolescents and Young Moroccan Adults
}

Ghizlane Ayach*, Habib El-Filali, Samia Saidi, Salma El-Gharib, Ghizlane Kaobai, Hasna Noaur, Selma El-Khayat, Mohamed Zamed, Ghizlane Medkouri, Khadija Hachim, Mohamed Benghanem Gharbi, Benyounes Ramdani

Department of nephrology, UCH Casablanca, Morocco

\begin{abstract}
Introduction: The primary nephrotic syndrome (PNS) is the most common glomerular nephropathy in children. Its diagnosis and management don't require histopathological study. It occurs mainly in the form of minimal glomerular lesion and in most cases respond to corticosteroids. The literature on histological lesions of pure PNS in adolescents and young adults is rare. Thus, there are no criteria or recommendations regarding the indications for renal biopsy in patients aged 12-18 years.

Methods: This is a retrospective study in which we encountered a total of 386 patients aged 12 to 25 years who were admitted and biopsied at the Nephrology Unit of Ibn Roshd Hospital in Casablanca during the period from January 1st, 2000 to September 30th, 2009 . Patients with pure PNS were 77 (20\%), all were included in this study.
\end{abstract}

Results: The average incidence of pure PNS was 7.7 cases per year. The study included 47 males (61\%) and 30 females (39\%). Patients were sent from all parts of Morocco and the average length of hospital stay was four days. Renal biopsies showed the following morphological lesions: minimal glomerular lesions in 61 cases $(79.20 \%)$, focal segmental hyalinosis in 7 cases $(9.10 \%)$, extramembranous glomerulonephritis in 7 cases $(9.10 \%)$ and 2 cases of renal amyloidosis $(2.6 \%)$.

Conclusion: The minimal glomerular lesions were the most common cause of pure primary nephrotic syndrome in patients aged 12-25 years. Initial renal biopsy may not be indicated in this age group, and an empiric therapeutic trial with corticosteroids may be initially considered.

* Corresponding Author; AYACH Ghizlane, hay El Farah II, rue Mohamed choukri NR 6 Settat, Morocco; E mail: ayachghizlane@ hotmail.fr
Keywords: Glomerular Disease; Children; Nephrotic Syndrome; Renal Biopsy

\section{The authors declared no conflict of interest}

\section{Introduction}

The primary nephrotic syndrome (PNS) is the most common glomerular nephropathy in children. Its diagnosis and management don't require a histopathological study. It occurs mainly in the form of minimal glomerular lesion and in most cases responds to corticosteroids. This was well demonstrated by the International Study of Kidney Disease in Children (ISKDC) in 1978 [1]. Therefore, renal biopsy was abandoned in children aged 2 to 12 years and the therapeutic approach with nephrotic syndrome is based on those studies $[1,2]$.

Several studies have been performed concerning clinical and therapeutic profiles in children and adolescents aged 12-18 years; however, the literature on histological lesions of pure PNS in this age group is rare. There are no criteria or recommendations regarding the indications for renal biopsy in patients aged 12-18 years presenting with pure PNS. The aim of the current study is to analyze the histological data of renal biopsies performed in patients aged 12-25 years and discuss the relevance and contribution of the renal biopsy in this patient population.

\section{Methods}

This is a retrospective study in which we encountered a total of 386 patients aged 12 to 25 years who were admitted and biopsied at the Nephrology Unit of Ibn Roshd Hospital in Casablanca during the period from January $1^{\text {st }}, 2000$ to September $30^{\text {th }}, 2009$. Patients with pure PNS were 77 (20\%), all were included in the study. Nephrotic syndrome was defined by proteinuria over 3 
Table 1: Distribution of histological lesions according to the age group in the study population

\begin{tabular}{lcccccc}
\hline & \multicolumn{2}{c}{$\mathbf{1 2}$ to 18 years } & \multicolumn{2}{c}{$\mathbf{1 9}$ to 25 years } & \multicolumn{2}{c}{ 12 to 25 years } \\
\cline { 2 - 7 } & Number & Percentage & Number & Percentage & Number & Percentage \\
\hline MGL & 27 & 79.40 & 34 & 79.10 & 61 & 79.20 \\
FSH & 4 & 11.76 & 3 & 6.98 & 7 & 9.10 \\
EMG & 2 & 5.88 & 5 & 11.63 & 7 & 9.10 \\
Amyloidosis & 1 & 2.94 & 1 & 2.32 & 2 & 2.60 \\
\hline
\end{tabular}

MGL: minimal glomerular lesions;FSH: focal segmental hyalinosis; EMG: extramembranous glomerulonephritis

Table 2: Comparison between the incidences of different nephropathies in the current study and the literature

\begin{tabular}{lccc}
\hline & ISKDC, $\mathbf{1 9 7 8}[\mathbf{1}]$ & Filler et al, 2003 [5] & Current series, 2009 \\
\hline MGL & $363(77.1 \%)$ & $115(72.3 \%)$ & $61(79.2 \%)$ \\
FSH & $37(7.9 \%)$ & $29(18.2 \%)$ & $7(9.1 \%)$ \\
EMG & $6(1.3 \%)$ & $3(1.9 \%)$ & $7(9.1 \%)$ \\
Others & $65(13.8 \%)$ & $12(7.5 \%)$ & $2(2.6 \%)$ \\
Total & $471(100 \%)$ & $159(100 \%)$ & $77(100 \%)$ \\
\hline
\end{tabular}

Data presented as number (percentage)

MGL: minimal glomerular lesions; FSH: focal segmental hyalinosis; EMG: extramembranous glomerulonephritis

$\mathrm{g} / 24$ hours, serum albumin below $3 \mathrm{~g} / \mathrm{dl}$ and serum protein less than $6 \mathrm{~g} / \mathrm{dl}$. Nephrotic syndrome was considered primary in the absence of macroscopic or microscopic hematuria, high blood pressure or renal impairment. Patients had to demonstrate the absence of signs of extrarenal involvement as well as negative serological and immunological tests. Clinical features of the patients were recorded. Inclusion criteria were:

1. Age of 12-25 years with pure primary nephrotic syndrome (PNS).

2. Availability of histological reports of two renal core biopsy specimens (light microscopy and immunofluorescence).

\section{Results}

During the period of our study, we encountered a total of 386 patients aged 12 to 25 years who were admitted and biopsied. Patients with pure PNS were 77 (20\%), with an average incidence of 7.7 cases per year. The average age was 19 years (range 12 to 25 years). The 77 PNS patients included 47 males (61\%) and 30 females (39\%). The gender ratio was 1.57 in favour of males. Patients were sent from all parts of Morocco with 70\% coming from outside Casablanca. The average length of hospital stay was four days, ranging from 2-8 days depending on the health condition of each patient. The morphological lesions identified were as follows: minimal glomerular lesions in 61 cases $(79.20 \%)$, focal segmental hyalinosis in 7 cases $(9.10 \%)$, extramembranous glomerulonephritis in 7 cases $(9.10 \%)$ and 2 cases of renal amyloidosis $(2.6 \%)$ (Table-1).

There was a gradual increase in the incidence of the PNS between the beginning and end of the study (Figure-1). This trend may be explained by the annual assignment of new nephrologists in different regions of the country who identified and managed those cases. There was also a decline from the year 2004 to 2005; this coincides with the temporary closure of the unit for renewal. The distribution of different histological findings according to patient age and year of presentation are shown (Figures 2-3).

\section{Discussion}

In children, nephrotic syndrome is the most common form of kidney disease with a male predominance. It responds to corticosteroids in more than $95 \%$ of cases with a very low risk of progression to renal failure. The studies conducted between 1967 and 1978 by the International Study of Kidney Disease in Children (ISKDC) showed that minimal glomerular lesions were the most common finding in idiopathic nephrotic syndrome in children, 
Figure 1: Incidence of pure primary nephrotic syndrome in the different years of the study

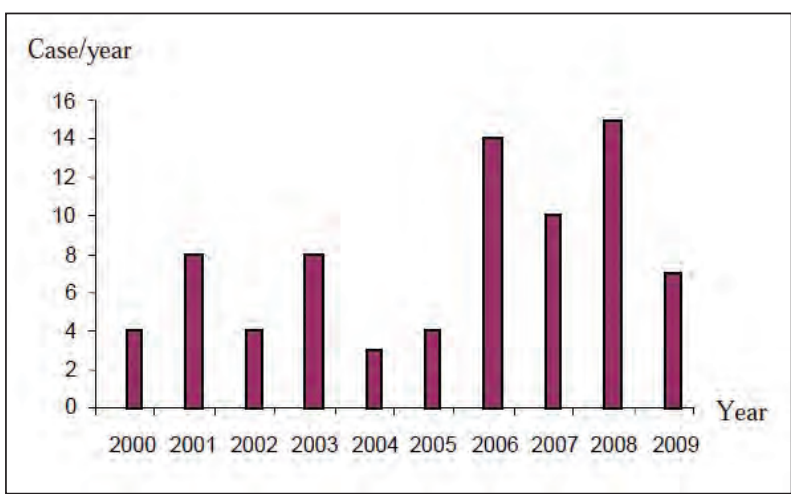

Figure 2: Incidence of different histological findings according to the patients age groups

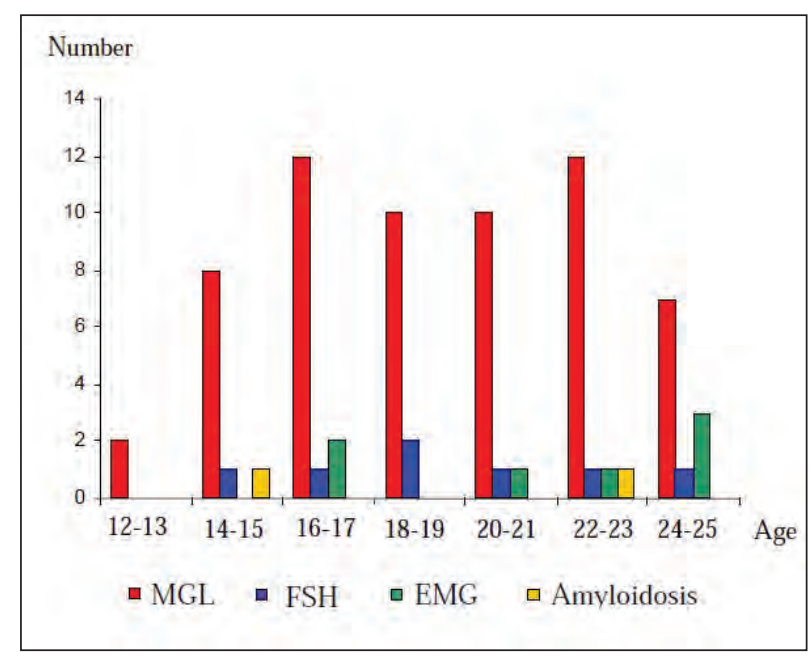

MGL: minimal glomerular lesions; FSH: focal segmental hyalinosis; EMG: extramembranous glomerulonephritis

representing $77 \%$ of biopsies compared to $8-10 \%$ in the case of focal segmental hyalinosis [1]. Among the 363 patients $(77 \%)$ who had minimal glomerular lesions, 338 (93.1\%) responded to corticosteroids. After the publication of these results, the routine practice of renal biopsy in this setting has been abandoned and limited to nephrotic syndrome associated with extrarenal signs or corticosteroid resistance after 28 days of treatment with prednisone at a dose of $60 \mathrm{mg} / \mathrm{m}^{2} /$ day.

For children over 12 years, there is no consensus on the value of histological study. Recently, several studies suggested an increase of other histological lesions in older children with nephrotic syndrome, particularly focal segmental hyalinosis [3-5]. Renal biopsy may be considered necessary for children over 12 years of age because of the possible high frequency of alternative diagnoses other than minimal glomerular lesions.

The nephrotic syndrome is a less common presentation of kidney disease in adults and almost always requires a renal biopsy to explore the etiological diagnosis. An important exception is the case of obvious long standing diabetes mellitus. Other causes include minimal change disease, extramembranous glomerulonephritis, focal and segmental glomerulosclerosis and amyloidosis.

Our results showed that minimal glomerular lesions were the major histopathological finding in this group of adolescents and young adults presenting with pure primary nephrotic syndrome. Minimal glomerular lesions were found in $79.2 \%$ of biopsies in contrast to $9.1 \%$ of biopsies that showed focal segmental hyalinosis (Table-1). These findings are largely similar to those reported by the ISKDC study group (Table-2). In addition, stratification of results according to age showed that all children below 14 years ( 13 cases) had minimal glomerular lesions, while extramembranous glomerulonephritis rate increased with age. These findings question the necessity of renal biopsy in patients aged 12 and 25 years presenting with PNS. Renal biopsy is an invasive procedure that is not without complications and requires hospitalization. From a health economics point of view, the procedure is expensive in our setting (travel, hospitalization expenses, laboratory tests, and biopsy needle costs). This is particularly relevant given that the basic medical needs are not covered for a third of the Moroccan population.

Besides its retrospective nature, an important limitation of our study is the fact that an electron microscopic (EM) documentation for minimal change disease was not carried out. EM is not widely available. Although early focal segmental glomerulosclerosis may present with mere foot process effacement, this lesion largely represents minimal change disease in the appropriate clinical setting.

\section{Conclusion}

Minimal glomerular lesions appear to represent the most common cause of pure primary nephrotic syndrome in patients aged 12-25 years in our setting. Primary diagnostic renal biopsy may not be necessary in this age group, and an empiric therapeutic trial with corticosteroids may be initially considered. Multicenter prospective studies are needed to verify our findings.

\section{References}

1. Nephrotic syndrome in children: prediction of histopathology from clinical and laboratory characteristics at time of diagnosis. A report of the International Study 
Figure 3: Incidence of different histological findings according to the year during the study period

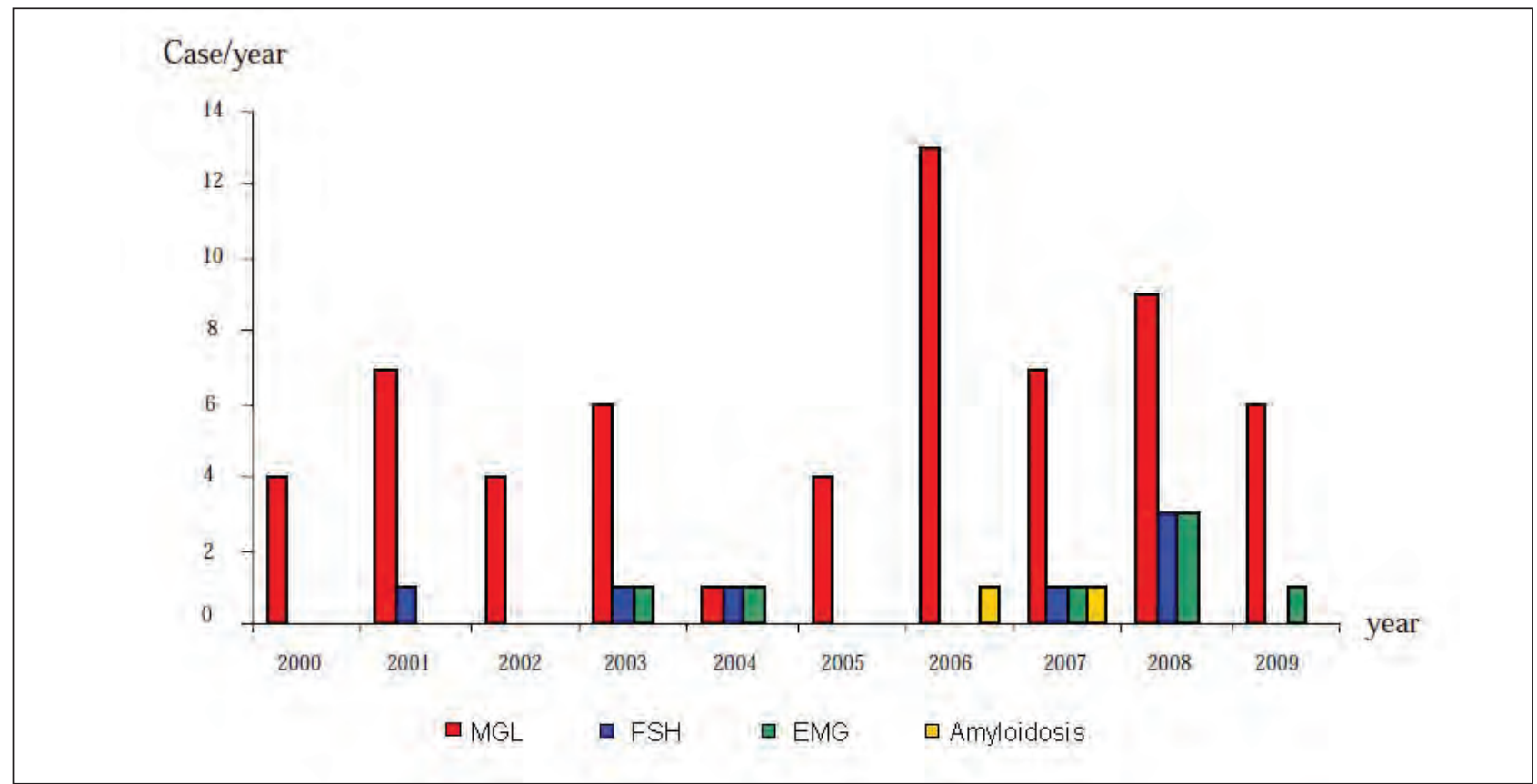

MGL: minimal glomerular lesions, FSH: focal segmental hyalinosis, EMG: extramembranous glomerulonephritis

of Kidney Disease in Children. Kidney Int. 1978 Feb;13(2):159-65.

2. Adu D. The nephrotic syndrome: does renal biopsy affect management? Nephrol Dial Transplant. 1996 Jan;11(1):12-4.

3. Borges FF, Shiraichi L, da Silva MP, Nishimoto EI, Nogueira PC. Is focal segmental glomerulosclerosis increasing in patients with nephrotic syndrome? Pediatr Nephrol. 2007 Sep;22(9):1309-13
4. Baqi N, Singh A, Balachandra S, Ahmad H, Nicastri A, Kytinski S, Homel P, Tejani A. The paucity of minimal change disease in adolescents with primary nephrotic syndrome. Pediatr Nephrol. 1998 Feb;12(2):105-7.

5. Filler G, Young E, Geier P, Carpenter B, Drukker A, Feber J. Is there really an increase in non-minimal change nephrotic syndrome in children? Am J Kidney Dis. 2003 Dec;42(6):1107-13. 